\title{
Put Something Back In On ne peut en retirer plus que ce qu'on y investit
}

In his outgoing address in President's Viewpoint, Harry Gairns wrote on the theme "What does the Institute do for me?"' Quite correctly he emphasized that the other side of the coin is represented by the question "What can I do for the Institute?". He also drew a parallel between the CIF/IFC and our country, Canada, when addressing these symbiotic questions.

I believe these intertwined themes are so important that I will attempt to advance them further on the thesis that in order to obtain optimal benefits from the Institute, from forestry, from the forests, or from Canada, we must be willing both individually and collectively to put something back in. The $\mathrm{CIF} / \mathrm{IFC}$ is a volunteer organization. As such it is highly dependent on personal commitment, initiative and sacrifice by its members in order to be successful. To a lesser, but still high degree, it is strengthened by the support of members employers and others in the forestry community.

Financial support, especially in recessionary times such as we have just come through, and which may continue yet a while in the forestry sector, may well be a significant personal or corporate sacrifice. Certainly it is an expense. But the real sacrifice made by our members is that of their time. After all, they must put something back into other components of their lives too. Beyond certain limits, time that members take away from their families or their work becomes dear indeed and at some point becomes an unreasonable sacrifice. While I encourage all forestry people to be members of the Institute, and all its members to actively contribute, I ask that they "live within their means" so to speak. It is not to the lasting benefit of the Institute or to forestry to have members set frantic, unsustainable paces for themselves.

The CIF/IFC does supply a medium for its members to contribute to forestry in ways that individually they could not. Our Sections for regional matters and our Working Groups and the Forest Science and Technology Board for sectoral ones are sound ways, together with the National Board of Directors, the National Executive, the Executive Director and direction from the annual general meeting to collectively address mutual concerns. By doing so the Institute and its members can both contribute and benefit at the same time.

We live in a time when a large and expanding human population places unprecedented demands upon our forests. These cannot be met without having an effect on the forest environment. It is increasingly demanded of our members and others in the forestry community that we meet society's needs for the forests with minimal adverse effects on the environment.

While it is not reasonable to think that the harvesting required to meet the current needs of our people can be done with negligible effect on the forest it is reasonable to believe that it can be done in ways that are ecologically more sound than have been some past practices.
Dans son dernier éditorial en tant que président, Harry Gairns a élaboré sur le thème de “Qu'est-ce que fait l'Institut pour moi?'" Il a souligné adéquatement que l'envers de la médaille était illustré par "Qu'est-ce que je peux faire pour l'Institut?". Il a aussi établi une comparaison entre l'IFC/CIF et notre pays, le Canada, en répondant à ces questions symbiotiques.

Je crois que ces deux thèmes si étroitement reliés sont tellement importants que je vais tenter de les amener plus loin dans la perspective que si nous voulons retirer les plus grands bénéfices de l'Institut, de la foresterie, des forêts, ou du Canada, nous devons être prêt, individuellement et collectivement à y investir. L'IFC/CIF est un organisme à adhésion volontaire. En tant que tel, il dépend grandement de l'engagement individuel, de l'initiative et de la dédication de ses membres de façon à être progressif. A un degré moidre, mais toujours aussi important, il est renforcé par l'appui des employeurs de ses membres et par les personnes qui composent la communauté forestière.

Le support financier, tout spécialement au cours d'une période de récession comme celle dont nous émergeons, et qui pourrait se poursuivre encore un certain temps dans le secteur forestier, pourrait bien constituer une implication personnelle ou corporative significative. Il s'agit bien d'une dépense. Mais la vrai dédication de nos membres réside dans le temps qu'ils consacrent à l'Institut. Après tout, ils se doivent de réinvestir aussi dans les autres composantes de leur vie. Au-delà d'une certaine limite, le temps que les membres empruntent à leur famille ou leur travail, devient coûteux dans les faits, et à un certain point cela constitue un sacrifice déraisonnable. Même si j'incite tous les gens en foresterie à devenir membre de l'Institut, et que tous ses membres y contribuent activement, je propose qu'ils "puissent vivre selon leurs moyens", si on peut dire. Il ne s'agit pas d'un bénéfice durable pour l'Institut ou pour la foresterie de voir ses membres maintenir un rythme frénétique et insoutenable.

L'IFC/CIF ne constitue pas un medium pour ses membres pour qu'ils contribuent à la foresterie de façon telle qu'individuellement ils n'auraient pu. Nos Sections pour les questions régionales, ainsi que les Groupes de travail et le Comité des sciences et technologies forestières pour les questions sectorielles sont des moyens éprouvés, en plus du Conseil d'administration, du Comité exécutif, du directeur exécutif et des résolutions émises par l'assemblée générale annuelle, pour collectivement répondre aux problèmes qui nous sont mutuels. En agissant ainsi, l'Institut et ses membres peuvent tous deux contribuer et en bénéficier par la même occasion.

Nous vivons à une époque où une importante et croissante population humaine exerce des demandes sans précédent sur nos forêts. Celles-ci ne peuvent être satisfaites sans provoquer d'effet sur l'environnement forestier. On demande de plus en plus à nos membres et à la communauté forestière de 
The active involvement of CIF/IFC in developing National Codes of Professional Forest Practice is one way to address this problem, and one in which the Institute is able to contribute more effectively than could individuals. The Institute's role in the national accreditation of professional forestry programs is another, as is our continued inpat into matters pertaining to sustainable development, a national forest strategy, forestry education and proper forest inventories.

Personally, I believe that we must always work towards improving the practice of forestry while recognizing that progress will sometimes be slow. We must not become discouraged by this. We must continue to advance from earlier achievements, building on strengths and eliminating weaknesses.

As your President 1 know that I will probably not achieve all that I would like to, but I will do the best I can. Please do the same. In order to get something out we must all put something back in.

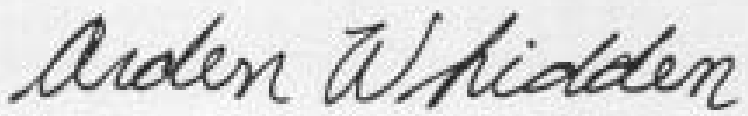

Arden Whidden, R.P.F., President satisfaire les besoins que la société attend de la forêt en minimisant les effets négatifs sur l'environnement. Même s'il n'est peu raisonnable de penser que l'exploitation forestière nécessaire pour combler nos besoins actuels peut étre faite sans effet néfaste sur les fort̀ts, il est raisonnable de eroire que l'exploitation peut être réaliste selon des méthodes qui sont plus écologiquement éprouvées que quelques-unes des pratiques antéricures.

La participation active de I'IFC/CIF dans l'élaboration des Codes nationaux de pratique professionnelle de la foresterie est l'une des façons de faire face à ce problème, ainsi qu'un moyen où I'Institut peut contribuer plus effectivement que ne pourrait le faire des individus. Le rôle de l'Institut au sein de l'équivalence nationale des programmes de foresterie professionnelle constitue un autre apport soutenu dans les domaines du développement durable, d'une stratégie forestière nationale, de la formation en foresterie et des inventaire forestiers adéquats.

Je crois personnellement que nous devons toujours travailler a l'amélioration de la pratique de la foresterie tout en reconnaissant que le progrès sera parfois lent à s'établir. Nous ne devons pas nous décourager. Nous devons poursuivre notre progression à partir des nos réalisations précédentes, en comptant sur nos forces et en remédiant à nos faiblesses.

En tant que président, je sais que fort probablement je ne pournai réaliser tout ce que $j$ 'aurais souhaité, mais je ferai de mon mieux. Tächez d'en faire autant. Si nous désirons en retirer quelque chose, nous devons tous y investir.

\section{Membership Dues 1991-1992}

Aetive Membership
Ist and 2 nd year after graduation
(grad 1990 , grad 1991 )
Other new members for first year
Married or equivalent, second member only
All other active members
Other Membernhips
Mernbers having retired status, prior to 1990-91
who wish to receive the Forestry Chronicle
Members having retired status, prior to $1990-91$,
who do not wish to roceive the
Forestry Chronicle
Members granted retirement status subsequent
to $1989-90$
Student Members
Sastaining Individuals (CIF Members)
Sustaining Corporate

\section{National Dues}

$\$ 82.00$
$\$ 82.00$
$\$ 82.00$
$\$ 114.00$

S 36.00

S 0.00

536.00

\$ 36.00

S 38.00

$\$ 240.00$

\section{Section Dues}

AL $\$ 8.00$
CC $\$ 8.00$
CH $\$ 8.00$
CO $\$ 10.00$
CR $\$ 8.00$
LW $\$ 13.00$
MB $\$ 10.00$
MR $\$ 00.00$
ND $\$ 10.00$
NO $\$ 5.00$
NS $\$ 10.00$

\author{
NW $\$ 12.00$ \\ Students $\$ \$ .00$ \\ OK $\$ \$ .00$ \\ OR $\$ 7.00$ \\ OV $\$ 16.00$ \\ PA $\$ 8.00$ \\ RM $\$ 10.00$ \\ SK $\$ 10.00$ \\ SN $\$ 8.00$ \\ SO $\$ 20.00$ \\ Students $\$ 20.00$ \\ VA $\$ 10.00$ \\ VI $\$ 5.00$
}

Upon written application, spousal members of Active Members, may have the dues of one of them reduced by an amount determined annually. Spousal members are members of the same Section who are fiving together as spouses and using the same mailing address.

The Instituse year runs from July 1 to June 30. Applications dated after January 1 will be charged half does for that Institute year. Applications dated after April 1 will be charged no dues for that Institute year. 